\title{
Comparison of the reported intakes of fruits and vegetables in Year 6 children: The CHANGE! Project
}

\author{
G. L. Warburton ${ }^{1}$, J. C. Abayomi ${ }^{1}$, E. Mahon ${ }^{1}$, R. Gobbi ${ }^{3}$, K. Mackintosh ${ }^{4}$, S. J. Fairclough ${ }^{2}$, \\ L. M. Boddy ${ }^{2}$, K. George ${ }^{2}$ and I. G. Davies ${ }^{1}$ \\ ${ }^{1}$ Faculty of Education, Community and Leisure, Liverpool John Moores University, IM Marsh Campus, Barkhill Road, \\ Liverpool L17 6BD, ${ }^{2}$ Research Institute for Sports and Exercise Sciences, Liverpool John Moores University, Liverpool, \\ L3 3AF, ${ }^{3}$ Faculty of Science and Social Science, Liverpool Hope University, Liverpool L16 9JD and ${ }^{4}$ Applied Sports \\ Technology Exercise and Medicine Research Centre (A-STEM), College of Engineering, Swansea University, \\ Swansea SA2 8PP, UK
}

The consumption of fruits and vegetables (FV) is considered an important part of a varied and balanced diet and ' 5 a day' is actively encouraged in Best Practice Guidance documents ${ }^{(1)}$. An intake of 5 or more portions of FV a day, can potentially contribute to a decreased risk for several chronic, lifestyle-associated diseases, for example, cardiovascular disease ${ }^{(2)}$, and type 2 diabetes $^{(3)}$. The CHANGE! Project (Children's Health, Activity, and Nutrition: Get Educated!), involving Year 6 children ( $n=290$ total; $n=138$ intervention; $n=152$ control) in Wigan, aimed to promote the consumption of FV as part of a healthy lifestyle. A teaching resource was adapted and Anglicised from the Planet Health programme ${ }^{(4)}$ and delivered by the teachers in the intervention schools. Data regarding the food intake of the participants was collected at three data collection points throughout the academic year, using a validated 24-hour recall questionnaire ${ }^{(5)}$. Participants were also asked to recall how many portions of FV they had consumed over the previous 24 hour period. Overall, fruit was more likely to have been purportedly consumed in the previous 24 hours than vegetables (Table). There were no significant differences between the control or intervention groups when tested for reported intakes of FV in the previous 24-hour period at any of the data collection points (Mann-Whitney test; $p \geq 0.194$ ).

\begin{tabular}{lccc}
\hline FRUITS - 'YES' $(\%)$ & Baseline & Post Intervention & Follow Up \\
\hline Control & 67.3 & 76.0 & 68.2 \\
Intervention & 74.4 & 74.4 & 74.8 \\
& & & \\
\hline VEGETABLES - 'YES' (\%) & Baseline & Post Intervention & Follow Up \\
\hline Control & 48.0 & 44.3 & 36.9 \\
Intervention & 51.9 & 47.8 & 37.5 \\
\hline
\end{tabular}

Whilst there was a significant difference (Mann-Whitney test, $p \leq 0.045$ ) between the reported numbers of portions of FV consumed at baseline, with the intervention group reportedly consuming approximately 1 more portion of both FV than the control group, there was no overall intervention effect. Mean intakes were between 2 and 3 portions in both groups. Although the intervention appeared to not significantly increase the frequency of intakes and/or number of portions consumed, it demonstrates that the reported intakes for approximately $70 \%$ of these children were in accordance with Government figures for households in the UK of 2.8-3.0 portions a day for girls and boys aged 11 years $^{(1)}$. Future research should focus on increasing FV to government targets of 5 or more per day.

1. Department of Health. Choosing a Better Diet: a food and health action plan. 2005 [updated 09/03/2005]; Available from: http://www.dh.gov.uk/en/ Publicationsandstatistics/Publications/PublicationsPolicyAndGuidance/DH_4105356.

2. Nikolic M, Nickic D, Petrovic B (2008) Fruit and vegetable intake and the risk for developing coronary heart disease. Cent Eur J Public Health 16, $17-20$.

3. Carter P, Gray LJ, Troughton J, Khunti K, Davies MJ (2010) Fruit and vegetable intake and incidence of type 2 diabetes mellitus: Systematic review and meta-analysis. BMJ 341, c4229.

4. Cheung L, Dart H, Kalin S, Gortmaker SL, editors. (2007) Eat Well and Keep Moving. 2nd ed. Champaign, IL: Human Kinetics.

5. Johnson B, Hackett A, Roundfield M, Coufopoulos A (2001) An investigation of the validity and reliability of a food intake questionnaire. J Hum Nutr Diet 14, 457-65. 